\title{
Recent Advances in Sire Evaluation Methods: A Review
}

\author{
Amol Jagannath Talokar, Harshit Kumar, Arnav Mehrotra, Kaiho Kaisa,
} K.A. Saravanan, Manjit Panigrahi, Triveni Dutt, Bharat Bhushan

10.18805/IJAR.B-4280

\begin{abstract}
This review deals with the various traditional and recent methods of sire evaluation. A sire evaluation is a method of prediction of sire's next-generation produced by breeding with specified females and creating their records in a specific environment. Breeding worth of progeny tested sires are obtained through sire index method that assigns ranking to each sire based on their genetic merit. Numerous sire indices are broadly classified in two type's viz., indices which are purely meant for ranking purposes and those which, besides, provide an estimate of the breeding worth of each sire. Sires can be evaluated in single or multiple herds. The statistical equations represent sire evaluation as linear or non-linear methods. Numerous methods can be incorporated as the advances are made in sire evaluation based on data structures, breeding approaches and selection methods. There are various approaches of sire evaluation such as Least Squares Method (LSM), Simple Regressed Least Squares (SRLS), Best Linear Unbiased Prediction (BLUP) and Derivative-Free Restricted Maximum Likelihood (DFREML) for single as well as multiple trait models which can be used to derive genetic worth of an individual. An efficient method of sire evaluation shows minimum within sire variance or error variance.
\end{abstract}

Key words: Bayesian estimation, GBLUP, Genomic selection, Prediction, Sire.

Sire evaluation can be defined as the process of selection of breeding bulls to be the future sires for genetic improvement based on the performance of their progeny and relatives (Henderson, 1973). Evaluation of sires for their ability to transmit economic traits to their daughters has gained significant importance because, in males, high selection intensity could be practiced (Smith and Banos, 1991). Besides, a larger impact can be seen in terms of more numbers of progeny the bulls can leave behind, production, reproduction and sex-limited traits that are not highly heritable (Naha et al. 2017). Further, the sire evaluation is more beneficial than dam evaluation because of more rigorous selection is possible in males, easy multiplication of elite germplasm and early selection of the males based on genetic markers (Peñagaricano et al. 2012). All of these aspects are cost as well as time-saving. Mostly, the sire evaluation is done in dairy cows and the genetic improvement depends on the accuracy of sire selection, selection intensity, generation interval and genetic variability of the traits considered (Hagiya, 2019).

Approximately, $61 \%$ of genetic gain in cattle derives from the selection of sires through two paths i.e. Bulls to breed cows and bulls to breed bulls (Robertson and Rendel, 1954). A sire is presumed to be more than half of the herd because the problems like non-conception risks and economics to maintain more numbers of dams are overcome by picking up a good serving bull. The use of artificial insemination (A.I.), multiple ovulation embryo transfer (MOET) and embryo transfer technology (ETT) at the field level shows exceptional results (Tan, 2002). The average life span of a typical bull is near about 6.29 years and the calf produced per bull depends upon the number of years worked along with the number of cows mated in a bull's lifetime (Statham et al. 2019). To validate a greater level of
Division of Animal Genetics, ICAR-Indian Veterinary Research Institute, Izatnagar, Bareilly-243 122, Uttar Pradesh, India.

Corresponding Author: Bharat Bhushan, Division of Animal Genetics, ICAR-Indian Veterinary Research Institute, Izatnagar, Bareilly-243 122, Uttar Pradesh, India.

Email: bhushan.drbharat@gmail.com

How to cite this article: Talokdar, A.J., Kumar, H., Mehrotra, A., Kaisa, K., Saravanan, K.A., Panigrahi, M., Dutt, T. and Bhushan, B. (2021). Recent Advances in Sire Evaluation Methods: A Review. Indian Journal of Animal Research. DOI: 10.18805/IJAR.B-4280.

Submitted: 13-08-2020 Accepted: 14-04-2021 Online: 29-04-2021

attainment in the genetic merit of a bull encompasses the multiplication of the dam to sire ratio with precise management, physical fitness and fertility of bull along with the cow's good body, normal cycling and disease free condition. Appropriate specification of a bull for the needs of a particular herd encouraged by using expected breeding values (EBVs) over the traditional method viz., phenotypic selection of sire. EBVs of the sire is a vital part of a longterm planned breeding approach and this approach will be more effective if recording and reporting systems in the developing countries advance to be precise to align reliability and accuracy of predictions for a bull's breeding efficiency (Laws, 2014 and Statham et al. 2019).

\section{History of sire evaluation}

Denmark was the first country to start evaluating dairy sires in 1902 (Kumar, 1984). Sire evaluation methods developed are linear as well as non-linear. Sire evaluation was initiated with the single herd in the case of dairy bulls, with subsequent expansion to multi-herd evaluation (Hancock et al. 2016). J. L. Lush elucidated principles of sire evaluation 
in 1931 and also classical selection problem in random samples from the population in animal breeding had principally been explained by Wright and Lush (Henderson, 1973; Hazel, 1943 and Lush, 1931). Rice (1944) proposed the 'New Index' using an equal parent index incorporating normal expectations and breed average. Varo worked on the evaluation of bulls from progeny testing in a different environment and concluded that relative evaluation methods based on deviation from herd average were more accurate to find the breeding value of sire for milk yield than the actual average yield of daughters (Kumar, 1984). Cunningham (1965) proposed a method of least squares for analysis of sire effect from non-orthogonal data and evaluation of sires at an early age. The selection approach in case of sire evaluation was also mixed model selection, in which the candidates for selection are randomly drawn from more than one population and the merit of each is the sum of the subpopulation mean and the value of the particular random variable associated with that animal (Henderson, 1973). Hazel with Lush established a selection index assumption to constitute the best utilization of the data (Hill, 2014). Smith (1936) had initiated selection index methods successfully in plant breeding and Hazel (1943) proposed this theory of genetic correlations and revealed how to use these to estimate multitrait selection indices in livestock and Lush (1947) decipher how to weight an index of records on an individual and its sibs.

\section{Linear methods for evaluation of sire}

\section{Least squares method (LSM) by Harvey (1960)}

The least-squares normal equations result from the use of a differential calculus principle and to construct this set of equations, there must be one equation for each of the constants to be estimated. The least-squares principle minimizes the error variance after adjusting the data for various non-genetic factors. The principle of LSM is based on the square of the difference between the observed and estimated value of the dependant variables must be least or zero. The estimation of the actual effect and prediction of the breeding values of sires were done sequentially in the population from statistical data (Hill, 2014).

Most important drawback of LSM is its high sensitivity to outliers (i.e., extreme observations). This is a consequence of using squares because squaring exaggerates the magnitude of differences (e.g., the difference between 20 and 10 is equal to 10 but the difference between $20^{2}$ and $10^{2}$ is equal to 300 ) and therefore gives a much stronger importance to extreme observations. This problem addressed by using robust techniques that are less sensitive to the effect of outliers. This field is currently under development and is likely to become more important in the next future.

\section{Restricted Maximum Likelihood Method by Patterson and Thompson (1971)}

Patterson and Thompson (1971) proposed a restricted maximum likelihood (REML) approach which consider the loss in degrees of freedom resulting from estimating fixed effects. The REML method is capable to yield unbiased estimates for variance components of the linear model (Patterson and Thompson, 1971). The principle of maximum likelihood estimation (MLE), initially developed by R.A. Fisher which expresses that the appropriate probability distribution is the one that yields the experimental data "most likely," which means that one must examine for the value of the parameter vector that utilizes the likelihood function. The MLE analyses the parameters by maximizing the logarithm of the likelihood function. The fact of maximum likelihood is comparable to the least-squares principle for ordinary linear regression. The maximum likelihood estimators are biased, therefore the MLE is improved by a method known as restricted maximum likelihood (REML) which removes bias in estimates and avoids negative estimates of a component of variance (Searle et al. 1992). Also, the REML is used to maximize a modified likelihood that is free of mean components instead of the inherent likelihood in a maximum likelihood. REML estimation is available in a number of general-purpose statistical software packages, including Genstat (the REML directive), SAS (the MIXED procedure), SPSS (the MIXED command), Stata (the mixed command), JMP (statistical software) and R (especially the Ime4 and older nIme packages) and in more specialist packages viz., MLwiN, HLM, ASReml, BLUPF90, wombat, Statistical Parametric Mapping and CropStat.

\section{Best Linear Unbiased Prediction (BLUP) by Henderson} (1973)

Charles Roy Henderson (1973) developed the most efficient method of sire evaluation. BLUP can be exploited with various models to predict breeding values of bulls and to evaluate non-genetic effects. The features of the BLUP method are as follows:

Best: Maximizations of the correlation between the true breeding values and predicted values or minimizes prediction error variance,

Linear: Predicted breeding values are a linear function of the observations,

Unbiased: Estimates of fixed effects are unbiased and are unknown, estimation of true breeding values for a random variable, such as sire's breeding values and

Prediction: it comprises the prediction of accurate breeding values.

Because of its desirable statistical properties, BLUP is largely used in genetic evaluation such as to predict sirebreeding values given measurements on progeny, breeding values of sires with repeated records, breeding values of all sires in the pedigree. Recently, this has been boosted by the steady increase in computing power and has advanced in terms of its application to sire model and then to more complex models, such as the animal, maternal, multivariate and random regression models (Mrode, 2014). BLUP was originally developed for ranking and selection in the contexts of animal breeding. It is an appropriate technique when the 
ideal ranking or selection criteria involve un-observable characteristics that may be regarded as random effects (Yadav et al. 2016).

BLUP sire model (BLUP-SM) is the simplest application of a BLUP for the prediction of breeding values that suggests only sires are being evaluated using progeny records in dairy cattle. The BLUP repeatability model is utilised for the analysis of data when multiple measurements on the same trait are recorded on an individual (Interbull, 2000). Whereas, the BLUP animal model (BLUP-AM) involves setting up equations for every animal, i.e., for all parents and progeny. The univariate model is simpler form of animal model assumes only additive genetic variance component of a phenotypic variable without repeated measurement of a trait across life of sire. The multivariate animal model analyzed two traits affected by both direct and maternal genetic effects. It is an extension of the univariate model and large sizes of the matrices involved in which the mixed model equation plays an important role in the analysis process. BLUP-AM revealed higher efficiency for sire evaluation in Indian buffalo for first lactation 305 days milk yield prediction as compared to least square and BLUP-SM (Sahoo et al. 2019).

\section{Genomic Best Linear Unbiased Prediction (GBLUP)}

Genomic Best Linear Unbiased Prediction (GBLUP) (Meuwissen et al. 2001) is more widely applicable than BLUP because it uses genomic relationships calculated from genome-wide SNP's and is applicable in populations with incomplete or missing pedigree information. In GBLUP, all genetic markers have equal weight. The model disregards the real genetic determinism of the trait and also the covariance between the genomic breeding values of two animals is proportional to their fraction of the genome they share (Boichard et al. 2016). GBLUP is specifically efficient for exact polygenic traits. The prior distribution of the GBLUP algorithm considers an equal variance over individual locus. The availability of compact DNA marker information has facilitated the large-scale genotyping of individuals for the prediction of sire's genetic merit. To forecast the disease risk and genetic merit, the SNPs which are abundant on the genome have been utilized for wide scopes in human, livestock and plant genetics. These markers are useful for the detection of areas of the genome that have a significant effect on quantitative trait variation, the prediction of an individual's risk to disease infection and the estimation of heritability, genetic variance components to determine the genetic value of individuals so that they can be selected for breeding purposes (Fernando et al. 2016).

\section{Recent advances in linear methods of sire evaluation methodology}

Derivative free restricted maximum likelihood (DFREML)

Meyer composed a software package that used derivativefree REML based on the likelihood ratio test to compare the significance of the variance components used in the model. DFREML supported 10 models that also included complex random regression. Now WOMBAT is the successor of DFREML for mixed model analysis using REML. Estimates of (co)variance components and heritability for breeding values and traits of the sire can be estimated by fitting a series of univariate animal models using a derivative-free REML algorithm. Permanent environmental and maternal genetic effects were considered by fitting random effects into the model of study.

Indian workers observed that the DFREML method was the most efficient and accurate for sire evaluation in Red Sindhi and Frieswal cattle using actual and predicted the first lactation 305-days milk yield. As per the results, it was suggested that the preference should be given to use the DFREML method followed by BLUP, SRLS and LSM methods for sire evaluation in Sahiwal (Mallick et al. 2018 and Rajeev et al. 2021).

\section{Average Information Restricted Maximum Likelihood Algorithm (AIREML)}

The restricted maximum likelihood (REML) method is frequently used to elucidate variance components and genetic parameters and it includes recognition of algorithms like expectation-maximization (EM) and the derivative-free (DF), whilst the 'average information' (AI) algorithm is recently attracting because of its desired computing properties. Precisely, AIREML is a quasi-Newton algorithm, which imposes first derivatives of the likelihood but replaces second derivatives with the average of the observed and expected information and it is computationally highly advantageous over derivative-free procedures.

The computing approach is limited to sparse matrix tools and programmed variation of a matrix that does not require the inversion of large, sparse matrices. When only one random factor and equal design matrices for all traits in the model, then to evaluate the likelihood, first and 'average' second derivatives can be carried out trait by trait, failing computational requirements of multivariate analysis to those of a series of univariate analyses (Meyer, 1997). This is eased by a canonical decomposition of the covariance matrices and analogous transformation of the data to new, uncorrelated traits. The rank of the predictable genetic covariance is resolute by the number of nonzero orthogonal matrix values of the canonical decomposition, as a result, it can be reduced by fixing several eigenvalues at zero. This limits the number of univariate analyses important to the required rank and is advantageous for the estimation of covariance function when a potentially large number of highly correlated traits are described by a low order polynomial. (Meyer, 1997).

FORTRAN programs for AIREML have been developed and a data set on different sires were analyzed to examine the relative computing properties. The AIREML does not use the predicted residuals also seems to be a useful numerical technique for the REML estimation with the individual animal model. Variance component approaches have been widely used to detect the existence of variation associated with 
quantitative trait loci (QTL). As a method to obtain REML estimates, the average information algorithm (AIREML) is used. The average information coefficients are the average of the observed and expected information matrices from the Newton Raphson and Fisher scoring method analyzed by iterative algorithm equation. An AIREML method was found appropriate for the fine-mapping of QTL.

\section{The Bayesian method in the sire evaluation}

Bayesian approach is a breakthrough improvement of robust and flexible computational algorithms in data science pioneered by Thomas Bayes in 1763 (Berger, 2000). Bayesian estimation or shrinkage estimation usually provide constant estimates with very few standard errors by allowing breeder prior information to be assimilated directly into the analysis. If the assumptions are encrypted in a probability distribution for the unknown parameters then this distribution is termed as the prior distribution. The data provides an unknown parameter that resulted from a sampling distribution. This inference of the analysis is the posterior distribution, a compromise between the prior information and the data information (Gianola, 2013). Bayesian methods recommended for the point estimates of the genetic and phenotypic parameters. There is clear scope for the use of Bayesian methods in sire selection and evaluation by treating in terms of a decision theory approach. This approach combines prior information on the parameters into the selection by constructing an index using posterior expectations of breeding values rather than parameter estimates. The Bayesian approach is then such as to maximize the posterior expected utility of the selection.

Prior information on the parameters would be included in the form of a prior probability distribution that takes advantage of the fact that the problem of selecting animals for breeding is essentially one of making the best decision. The use of the decision theory approach does not comprise the estimation of parameters and so the problem of nonsensical estimates does not arise. The Bayesian analysis involves choosing a parametric model for the data, assigning a prior distribution to the unknown parameters and then investigating the resulting joint posterior distribution. The prior distribution should accurately replicate the prior opinions of the animal breeders and the analysis of the posterior distribution should include sufficient marginal and conditional distributions to sufficiently define the entire function.

Gibbs sampling approach specifies output readily translated into prescribed assumption summaries may apply to combinations using relevant families of prior distributions. Gibbs sampling output is required to improve suitable graphical methods for point evaluations of posterior distributions of genetic and phenotypic parameters and to compute the posterior expectations of breeding values and the predictable progress using various selection procedures. Thus, Bayesian procedures give improved selection decisions as they make use of all the information on parameters rather than just providing point estimates.
Bayesian analysis of data involves treating all parameters, including the variance components, as random and finding the joint posterior distribution of all the parameters given in the data. Because the selection decision depends on the variance components value in a Bayesian analysis which can yield a different selection outcome as compared to traditional analysis.

Bayesian approach is developed in four different types for analysis model i.e. A) Bayes A, B) Bayes B, C) Bayes C and D) Bayes $C \pi$. In Bayes $A$, markers are supposed to have several variances and the conditional distribution of every result to monitor a normal distribution (Meuwissen et al. 2001). The variances are expected to follow a scaled inversed $\chi^{2}$ distribution with degrees of freedom $v_{a}$ and scale parameter $\sigma_{a}^{2}$. Bayes $A$ varies from ridge regression (RR)BLUP signifying that each SNP has its variance. A model can also be supposed with $\pi$ closer to one imitating that most sections of the genome do not contain markers linked with the trait. This model is termed Bayes $B$ by Meuwissen and coworker (Meuwissen et al. 2001). The prior in Bayes B presumes variance of markers as zero with probability $\pi$ and the complement with probability $(1-\pi)$ which follows an inverse $\chi^{2}$ distribution with $v$ degree of freedom and scale parameter $\sigma$. The meaning of the probability $\pi$ relies on the genetic architecture of the trait, representing progress to the Bayes B model, identified as Bayes $C \pi$. The effects of SNP have a common variance in Bayes $C \pi$, which pursue a scaled inverse $\chi^{2}$ prior with parameters va, $\sigma^{2}{ }_{a}$ (Habier et al. 2011). Thus, the outcome of an SNP fitted with probability $(1-\pi)$ leads to a mixture of multivariate Student's $t$ distributions, $t\left(0, v_{\mathrm{a}}, / \sigma_{\mathrm{a}}^{2}\right)$, where, $\pi$ is the probability of a marker having zero effect. Parameters va and $\sigma^{2}$ are preferred as described for Bayes $A$. The $\pi$ parameter is treated as unidentified with a uniform $(0,1)$ prior distribution. Bayes $C$ utilizes a common variance for all SNPs. Bayes $\mathrm{Cp}$ (Habier et al. 2011) are the conversion of Bayes $C$ where the probability of possessing a zero effect SNPs is predicted.

\section{Multiple-trait Across Country Evaluation (MACE) Program of Interbull}

The International Committee for Animal Recording (ICAR) is the solitary organization for the standardization of animal recording and genetic evaluation globally. The organization (ICAR) was founded in 1951 and INTERBULL became a permanent sub-committee that supported it during 1988. The establishment of a global market in dairy cattle breeding made it essential to develop a single system for comparing sire evaluations across different countries. Sweden in 1983, precisely led to the foundation of INTERBULL to establish a set of recommendations for the construction of conversion formulae and to introduce improved prediction methods. In 1995, INTERBULL acquainted MACE and it was accepted in the UK for Jersey, Guernsey and Ayrshire breed in 1997 and Holstein during 2001. INTERBULL carries out MACE in two stages; 1) each participating country must submit its national evaluations and 2) This will be combined to provide 
each country with the outcomes of all sires expressed on its genetic base and scale. Interbull estimates bulls of six breeds and seven trait groups (milk production, udder health, conformation, longevity, calving, female fertility and drought ability traits). Other traits are going to be included in the future. Details of the MACE program depend on the sire reranking between some countries when these sire's performance is superior in certain environments than in others or when genetic evaluation approaches vary countrywise. Thus, it is required to calculate a separate set of results for each participating country by MACE. Since 2003 the Steering Committee is determined by two skilled groups and having nine members; the Scientific Advisory Committee (SAC) and the Interbull Technical Committee (ITC). The SAC aims to suggest methodological progress that is necessary to ensure the strategic direction, scientific soundness and long-term advancement of the Interbull services. The mandate of the ITC is to identify and review technical issues that may be necessary for providing a high-quality service to nations collaborating in the international genetic evaluation (http://www.interbull.org/ib/linkicar).

\section{Advances at the molecular and genomic level for sire evaluation}

\section{Marker-assisted selection}

With the advent of molecular markers and marker-assisted selection (MAS), the promise of improvement in the performance of bull is high. With MAS, the selection of better sire (with improved performance for economic traits) would be based on molecular markers (RFLP, microsatellite, SNP, etc.) that are genetically linked to the genes of interest affecting trait(s) of economic importance. The progress of MAS generally depends on the existence of linkage disequilibrium (LD) between specific gene or quantitative trait loci (QTL) and the DNA marker. However, the applicability of genetic markers and MAS in recent years is limited due to varied reasons such as complexity of production traits, polygenic inheritance, inadequate marker coverage, absence of LD between markers and economic trait, improper phenotype recording and complexity of solving equations that link phenotypic and molecular data (Boichard et al. 2016).

\section{Marker-assisted introgression}

Marker-assisted introgression (MAI) is one of the most important applications of molecular information in animal breeding targeting at introgression of one or more favorable genes from a donor to recipient with the least change in the genetic background of the recipient. It comprises of three segments 1) cross between donor and recipient lines to receive $F 1$; 2) repeated backcrossing with the $F 1$ and the subsequent progenies with the recipient to regain its genetic background and 3) intercross the backcrossed progenies to set introgressed genes in the population. Many features affect the efficacy of MAI, among which the methods of foreground and background selection are very critical.
Marker or Genomic-assisted introgression could eventually be used to introduce favorable alleles found in the elite sire into more widely used breeds (Groeneveld et al. 2016).

Good maps of molecular markers now available for many species and these maps are utilized in commercial breeding programs. This approach is suitable for the populations resulted from inbred line crosses and outbred populations specifically in the case of dairy cattle. It is believed that collective marker information into breeding programs can improve response to selection when the importance of MAl and marker typing costs are controlled to very little (Whittaker, 2004).

\section{Genomic selection (GS)}

Meuwissen with Goddard and Hayes was the first to proposed genomic selection in 2001. Genomic selection is a recent technology in cattle that includes selection based on (GEBV) genomic breeding values. The GEBV are constructed based on the marker-assisted selection in which genetic markers or haplotypes throughout the complete genome covering all quantitative trait loci (QTL) contributing to the variation in a trait or linkage disequilibrium with at least one marker (Hayes et al. 2009). The best method to evaluate the breeding value from genomic data is to calculate the effect of each QTL determined from the haplotypes or SNPS in a large reference population with phenotypic information. As a result, only marker information is required to calculate GEBV in succeeding generations (Gianola, 2013).

The revolutions which ensued in genomic selection methodology from the identification of thousands of SNP markers and SNP-chip genotyping tools that accounts for the genotyping of all these SNPs as a cost-effective (Meuwissen et al. 2016). In GS, the sum effect of all the SNPs is predicted at once without any significant study as compared to MAS, where the least number of significant markers were used and the other was treated as having zero effect (Meuwissen et al. 2016). The SNP-chip genotyping tools designed by Illumina and Affymetrix were confined to 50K SNPs for cattle. An advancement like wholegenome sequencing in many livestock species, high accuracy of GEBV prediction is attainable through the amalgamation of whole-genome sequence data, prominent sample sizes besides a statistical method analyzing the informative polymorphisms (Eggen, 2012).

The genotypic information and genomic data analysis is used for making predictions that are important for making informed decisions related to animal breeding. Thus, the regular collection of genomic data would be an important resource for the efficient management of breeding programs. The technical and economic feasibility of animal breeding programs has significantly increased after the use of genomic selection (Kariuki et al. 2017). The two pivotal expected advantages of applying GS are reduction in generation interval and increased accuracy of EBV (Shumbusho et al. 2016). With the acceleration of selection 
cycles, GS offers an opportunity to increase the selection gains (response) per unit of time (Meuwissen et al. 2001). Genomic selection allows the identification of genetically superior sires at a much earlier age. The valuable genomic information may predict the genetic merit of young animals with up to double the accuracy of traditional parent averages.

\section{SUMMARY AND CONCLUSION}

Sire evaluation was started from the 1900 s to deliver an independent comparison of the breeding performance of sires by evaluating their progeny in comparison to the progeny of other sires. A sire's superiority is usually centered on its genetic merit ranking. Initially, sire evaluations were correlated with phenotypic performances. With the advancement of mixed model methods, genetic evaluations providing more precise estimates of breeding value. Earlier through the sires model which considered sire-progeny relationships, then through the animal model, which considered all known relationships among animals in the pedigree. Expending this methodology, it is possible to estimate the fixed effects by BLUE and random effects by BLUP. Therefore, the BLUP answer is acquired for all animals present in the pedigree. This approach precisely yields estimated breeding values which are mostly acknowledged as a selection tool in sire evaluation. DFREML assumes the maternal genetic or permanent environmental effects to assess the heritability and (co)variance components for breeding values and traits of the sire and it is most preferred method rather than BLUP, SRLS and LSM. During 2001, GBLUP uses genomic relationships calculated from genome-wide SNP markers and is applicable in populations with incomplete or missing pedigree information. An AIREML method was found appropriate for the finemapping of QTL. The robust computational algorithms like Bayesian methods give more stable estimates with smaller standard errors by allowing breeders prior information to be assimilated directly into the analysis. The global market in dairy cattle breeding for comparing sire evaluations across different countries led to the formation of INTERBULL is a gold standard in bull evaluation.

Recently, the MAS was practiced in dairy herd established on phenotypic information for the prior selection of sire to reduce generation intervals and increase accuracy in progeny testing programs globally. Other molecular substitutes are being extensively deliberated such as GS because SNPs are the most abundant DNA polymorphisms in an entire genome and they have more privileged because of their ability to read genotypes automatically and very fewer mutation rates. GS provides (1) more accurate predictions of breeding values, particularly for traits having low heritability and that are expressed in one sex, (2) lower rates of inbreeding in the case of family selection in bull, (3) prior selection of sire in the case of slaughter traits and (4) facilitate the estimation of the traits of high cost or difficult to measure.
The major genomic estimation methods are GBLUP, Bayes A, Bayes B, Bayes C and Bayes C $\pi$. The accuracy and stability of the BLUP-AM observed are highest. To accelerate genetic gain, genomic selection should be integrated with the progeny testing (PT) programs. There is a need for the strengthening of the germplasm collection and dissemination network to evaluate dairy sires. BayesB is among the gold standards for evaluating new approaches, while a modified implementation of the BLUP approach GBLUP is used in many practical applications of genomic selection arranging a coherent framework to determine many applied complications in sire evaluation.

\section{REFERENCES}

Berger, J.O. (2000). Bayesian analysis: A look at today and thoughts of tomorrow. Journal of the American Statistical Association. 95(452): 1269-1276.

Boichard, D., Ducrocq, V., Croiseau, P. and Fritz, S. (2016). Genomic selection in domestic animals: Principles, applications and perspectives. Comptes rendus biologies. 339(7-8): 274-277.

Cunningham, E.P. (1965). The evaluation of sires from progeny test data. Animal Science. 7(2): 221-231.

Eggen, A. (2012). The development and application of genomic selection as a new breeding paradigm. Animal Frontiers. 2(1):10-15.

Fernando, R.L., Cheng, H. and Garrick, D.J. (2016). An efficient exact method to obtain GBLUP and single-step GBLUP when the genomic relationship matrix is singular. Genetics Selection Evolution. 48(1): 80.

Gianola, D. (2013). Priors in whole-genome regression: The Bayesian alphabet returns. Genetics. 194(3): 573-596.

Groeneveld, L.F., Gregusson, S., Guldbrandtsen, B., Hiemstra, S.J., Hveem, K., Kantanen, J. and Berg, P. (2016). Domesticated animal biobanking: Land of opportunity. PLoS Biology. 14(7): e1002523.

Habier, D., Fernando, R.L., Kizilkaya, K. and Garrick, D.J. (2011). Extension of the Bayesian alphabet for genomic selection. BMC Bioinformatics. 12(1): 186.

Hagiya, K. (2019). Development of genetic evaluation for milk production traits of Holsteins in Japan. Animal Science Journal. 90(4): 457-461.

Hancock, A.S., Younis, P.J., Beggs, D.S., Mansell, P.D., Stevenson, M.A. and Pyman, M.F. (2016). An assessment of dairy herd bulls in southern Australia: 1. Management practices and bull breeding soundness evaluations. Journal of Dairy Science. 99(12): 9983-9997.

Harvey, W.R. (1960). Least-squares analysis of data with unequal subclass numbers. Agricultural Research Service, United States Department of Agriculture. (Vol. 20, No. 8).

Hayes, B.J., Bowman, P.J., Chamberlain, A.J. and Goddard, M.E. (2009). Invited review: Genomic selection in dairy cattle: Progress and challenges. Journal of Dairy Science. 92(2): 433-443.

Hazel, L.N. (1943). The genetic basis for constructing selection indexes. Genetics. 28(6): 476-490.

Henderson, C.R. (1973). Sire evaluation and genetic trends. Journal of Animal Science, 1973 (Symposium). 10-41. 
Hill, W.G. (2014). Applications of population genetics to animal breeding, from Wright, Fisher and Lush to genomic prediction. Genetics. 196(1): 1-16.

Interbull (2000). National Genetic Evaluation Programmes for Dairy Production Traits Practised in Interbull Member Countries 1999-2000. Department of Animal Breeding and Genetics, Uppsala, Sweden, Bulletin. 24.

Kariuki, C.M., Brascamp, E.W., Komen, H., Kahi, A.K. and Van Arendonk, J.A.M. (2017). Economic evaluation of progeny-testing and genomic selection schemes for small-sized nucleus dairy cattle breeding programs in developing countries. Journal of Dairy Science. 100(3): 2258-2268.

Kumar, B. (1984). Method of sire evaluation in buffaloes. (Doctoral dissertation, NDRI, Karnal).

Laws, D. (2014). Fit for purpose bulls. A blueprint for breeders.

Lush, J.L. (1947). Family merit and individual merit as bases for selection. Part I. The American Naturalist. 81(799): 241261.

Lush, J.L. (1931). The number of daughters necessary to prove a sire. Journal of Dairy Science. 14(3): 209-220.

Mallick, P.K., Ghosh, A.K. and Rajendiran, A.S. (2018). Sire evaluation using animal model versus different conventional methods in Red Sindhi cattle. Indian Journal of Animal Research. 52: 1-6.

Meuwissen, T., Hayes, B. and Goddard, M. (2016). Genomic selection: A paradigm shift in animal breeding. Animal Frontiers. 6(1): 6-14.

Meuwissen, T.H., Hayes, B.J. and Goddard, M.E. (2001). Prediction of total genetic value using genome-wide dense marker maps. Genetics. 157(4): 1819-1829.

Meyer, K. (1997). An'average information'restricted maximum likelihood algorithm for estimating reduced rank genetic covariance matrices or covariance functions for animal models with equal design matrices. Genetics Selection Evolution. 29(2): 97.

Mrode, R. (2014). Best linear unbiased prediction of breeding value: univariate models with one random effect. Linear models for the prediction of animal breeding values, (Ed. 3): 3460.

Naha, B.C., Chakravarty, A.K., Mir, M.A., Bhakat, M., Singh, A.P. and Maher, D. (2017). Evaluation of bull fertility of Sahiwal breeding bulls. Indian Journal of Animal Research. 51(4): 801-802.
Patterson, H.D. and Thompson, R. (1971). Recovery of inter-block information when block sizes are unequal. Biometrika. 58(3): 545-554.

Peñagaricano, F., Weigel, K.A. and Khatib, H. (2012). Genome wide association study identifies candidate markers for bull fertility in Holstein dairy cattle. Animal Genetics. 43: 65-71.

Rajeev, Kumar, R. and Singh, R. (2021). Sire evaluation based on first lactation 305 day milk yield and individual part lactation records in Frieswal cattle. Asian Journal of Dairy and Food Research. DOI: 10.18805/ajdfr.DR-1610.

Rice, V.A. (1944). A new method for indexing dairy bulls. Journal of Dairy Science. 27(11): 921-936.

Robertson, A. and Rendel, J. (1954). The performance of heifers got by artificial insemination. The Journal of Agricultural Science. 44(2): 184-192.

Sahoo, S.K., Singh, A., Ambhore, G.S., Dash, S.K. and Dubey, P.P. (2019). Comparative efficiency of different multiple linear regression prediction equations of first lactation 305-day milk yield for sire evaluation in Murrah buffaloes. Indian Journal of Animal Research. 53(10): 1287-1291.

Searle, S.R., Casella, G. and McCulloch, C.E. (1992). Variance components. John Wiley and Sons. Inc. New York.

Shumbusho, F., Raoul, J., Astruc, J.M., Palhiere, I., Lemarié, S., Fugeray-Scarbel, A. and Elsen, J.M. (2016). Economic evaluation of genomic selection in small ruminants: A sheep meat breeding program. Animal. 10(6): 1033-1041.

Smith, C. and Banos, G. (1991). Selection within and across populations in livestock improvement. Journal of Animal Science. 69(6): 2387-2394.

Smith, H.F. (1936). A discriminant function for plant selection. Annals of Eugenics. 7(3):240-250.

Statham, J., Burton, K. and Spilman, M. (2019). Looking after the bull: guide to management and assessment of fertility. In Practice. 41(2): 69-83.

Tan, E.S. (2002). 'The bull is half the herd': property rights and enclosures in England, 1750-1850. Explorations in Economic History. 39(4): 470-489.

Whittaker, J.C. (2004). Marker Assisted Selection and Introgression. Handbook of Statistical Genetics.

Yadav, A.S., Dhaka, S.S., Magotra, A. and Malik, A. (2016). Efficiency of sire evaluation methods by using phase and stayablity traits to improve milk yield of Murrah buffaloes. Indian Journal of Animal Research. 50: 851-855. http:// www.interbull.org/ib/linkicar. 